\title{
C-Reactive Protein Level as Diagnostic Marker in Young Febrile Children Presenting in a General Practice Out-of-Hours Service
}

\author{
Marijke Kool, GP, PhD, Gijs Elshout, GP, PhD, Bart W. Koes, PhD, \\ Arthur M. Bohnen, GP, PhD, and Marjolein Y. Berger, GP, PhD
}

Background: It is unclear how well a C-reactive protein (CRP) value predicts a serious infection (SI) in young febrile children in general practice.

Methods: This prospective cohort study with 1-week follow-up included children, aged 3 months to 6 years, presenting with fever to a general practitioner out-of-hours service. We evaluate whether CRP level has predictive value for diagnosing a child at risk for an SI either at presentation or during followup. The index test was CRP $\leq 20 \mathrm{mg} / \mathrm{L}$ (rule out an SI) and $>80 \mathrm{mg} / \mathrm{L}$ (rule in an SI). The reference standard was referral to a pediatric emergency department or diagnosis of an SI. The main outcome measure was CRP value.

Results: CRP level was available for 440 children. To rule out an SI, CRP $\leq 20 \mathrm{mg} / \mathrm{L}$ did not change the probability of having no SI $(87.5 \%)$. CRP $>80 \mathrm{mg} / \mathrm{L}$ increased the probability of having an SI from $11.4 \%$ (pretest probability) to $21.2 \%$ (posttest probability). In children without a diagnosis of SI at presentation, CRP could not predict an SI during follow-up (CRP >80 mg/L: positive likelihood ratio, 2.1, 95\% confidence interval, 1.3-3.5; CRP $\leq 20 \mathrm{mg} / \mathrm{L}$ : negative likelihood ratio, $0.9,95 \%$ confidence interval, 0.7-1.2).

Conclusions: In general practice CRP has little clinically relevant value in discriminating febrile children in need of medical care from those who are not. (J Am Board Fam Med 2016;29:460-468.)

Keywords: C-Reactive Protein, Child, Cohort Studies, Hospital Emergency Service, Family Practice, Fever, Follow-Up Studies, General Practice, General Practitioners, Hospital Records, Humans, Outcome Assessment (Health Care), Point-of-Care Systems, Probability, Prospective Studies, Referral and Consultation

Most young febrile children presenting in primary care suffer from self-limiting infectious diseases. Very few children develop a serious infection (SI) that might require an antibiotic prescription or hospital admission. ${ }^{1,2}$ So-called alarming signs or red flags are common in febrile children, and most common alarming signs hardly discriminate be-

This article was externally peer reviewed.

Submitted 22 September 2015; revised 1 April 2016; accepted 11 April 2016.

From the Department of General Practice, Erasmus Medical Center, University Medical Center Rotterdam, Rotterdam, the Netherlands (MK, GE, BWK, AMB, MYB); and the Department of General Practice, University of Groningen, University Medical Center Groningen, Groningen, the Netherlands (MYB).

Funding: This study was supported by the Netherlands Organisation for Health Research and Development (ZonMw; dossier 42000012). The authors were independent of the funders. The funders were not involved in the design of the study. tween children at high or low risk for an SI. Therefore febrile children account for a large part of the workload of general practitioners (GPs) at the outof-hours service (OHS). A simple point-of-care test with good discriminative power might make triage at the OHS more efficient and might prevent unnecessary antibiotic prescriptions. ${ }^{3}$

C-reactive protein (CRP) is an acute-phase protein with increased concentrations in the blood during infections. ${ }^{4} \mathrm{~A}$ high CRP concentration is of prognostic value for pneumonia in adults presenting to primary care ${ }^{5-7}$ and has moderate to good

Conflict of interest: none declared.

Corresponding author: Marjolein Y. Berger, GP, PhD, Department of General Practice, University of Groningen, University Medical Center Groningen, PO Box 196, 9700 AD Groningen, the Netherlands (E-mail: m.y.berger@ umcg.nl). 
test characteristics in febrile children visiting a pediatric emergency department. ${ }^{8,9}$

Although CRP concentration is often routinely measured in pediatric departments, testing for CRP has little influence on decision making. ${ }^{10}$ From that perspective, and based on a systematic review, it was suggested that different cutoff levels could be used to rule in or rule out SI in febrile children'; however, no study in the latter review was performed in a low-prevalence general practice setting.

Furthermore, all studies performed to date evaluated whether CRP predicts SI at presentation (cross-sectional design). No study has evaluated whether CRP predicts the development of an SI.

Using point-of-care tests, CRP concentrations are available within minutes ${ }^{11}$; moreover, an increasing number of GPs has the possibility to test CRP at the point of care. In Dutch primary care guidelines, however, CRP point-of-care tests are still not recommended for febrile children because of a lack of evidence regarding their diagnostic or prognostic value in this population. ${ }^{12,13}$

In this study we aim to evaluate whether the CRP concentration in young febrile children presenting to a general practice OHS has value for predicting an increased risk of SI at presentation or during 1-week follow-up.

\section{Methods}

\section{Design and Setting}

This prospective cohort study was performed at an OHS in the southern part of Rotterdam, the Netherlands. This OHS serves an area with 300,000 inhabitants. The study was approved by the Dutch Central Committee on Research Involving Human Subjects.

\section{Population}

Between December 2004 and January 2006, all consecutive children aged 3 months to 6 years whose parents called the OHS concerning the febrile child were eligible for inclusion. Fever (as reported by the parents) had to be a reason to contact the OHS. The child was excluded if parents could not communicate in Dutch, if there was no informed consent, or if the child had been enrolled in the study in the previous 2 weeks ${ }^{2,14}$ (Figure 1).

\section{Index Test}

Capillary blood was obtained as soon as possible, within 24 hours of inclusion. CRP was measured using the Nycocard CRP test (Clindia Diagnostics, Leusden, the Netherlands) directly after physical examination. The Nycocard CRP test is a pointof-care test that can be conducted within 5 minutes and correlates well with a reference test performed in the laboratory. ${ }^{11}$

GPs, pediatricians, and parents were not informed about the CRP value. In some children CRP concentration was not obtained for the purpose of the study because of referral to a hospital at the moment of CRP measurement. These children were included if a CRP concentration measured by the pediatrician was available. If children showed resistance to the finger prick, this was interpreted as no informed consent from the child and the fingerprick was not performed.

\section{Reference Standard}

"At risk for an SI" was our reference standard. We defined a child as being at risk for an SI when the child was referred to a pediatrician or was diagnosed with a serious illness at initial presentation or during follow-up. In addition, we defined "SI at presentation" when a child was referred or was diagnosed with a serious illness at presentation, and we defined "SI during follow-up" when the child was referred or was diagnosed with a serious illness during follow-up.

SIs included pneumonia, sepsis, meningitis, encephalitis, pyelonephritis, osteomyelitis, cellulitis, erysipelas, abscess, dehydration (caused by gastroenteritis or an unknown cause), asthma exacerbation with fever, and, in children aged $\leq 1$ year, bronchiolitis. The OHS records were examined for diagnostic codes registered according to the International Classification of Primary Care and noted by the GP. In children who had a consultation with their own GP during follow-up, that GP's records were examined for diagnostic codes. If a contact was not coded, a team of 3 GPs allocated an International Classification of Primary Care code based on the (uncoded) diagnosis made by the GP or, if this diagnosis was missing, based on noted symptoms and findings during the physical or history examination. The team was blinded to the triage result, CRP concentration, and management by the GP. Final coding was based on consensus. ${ }^{2,14}$ 
Figure 1. Flowchart of inclusion of all eligible children. CRP, C-reactive protein; OHS, out-of-hours service.

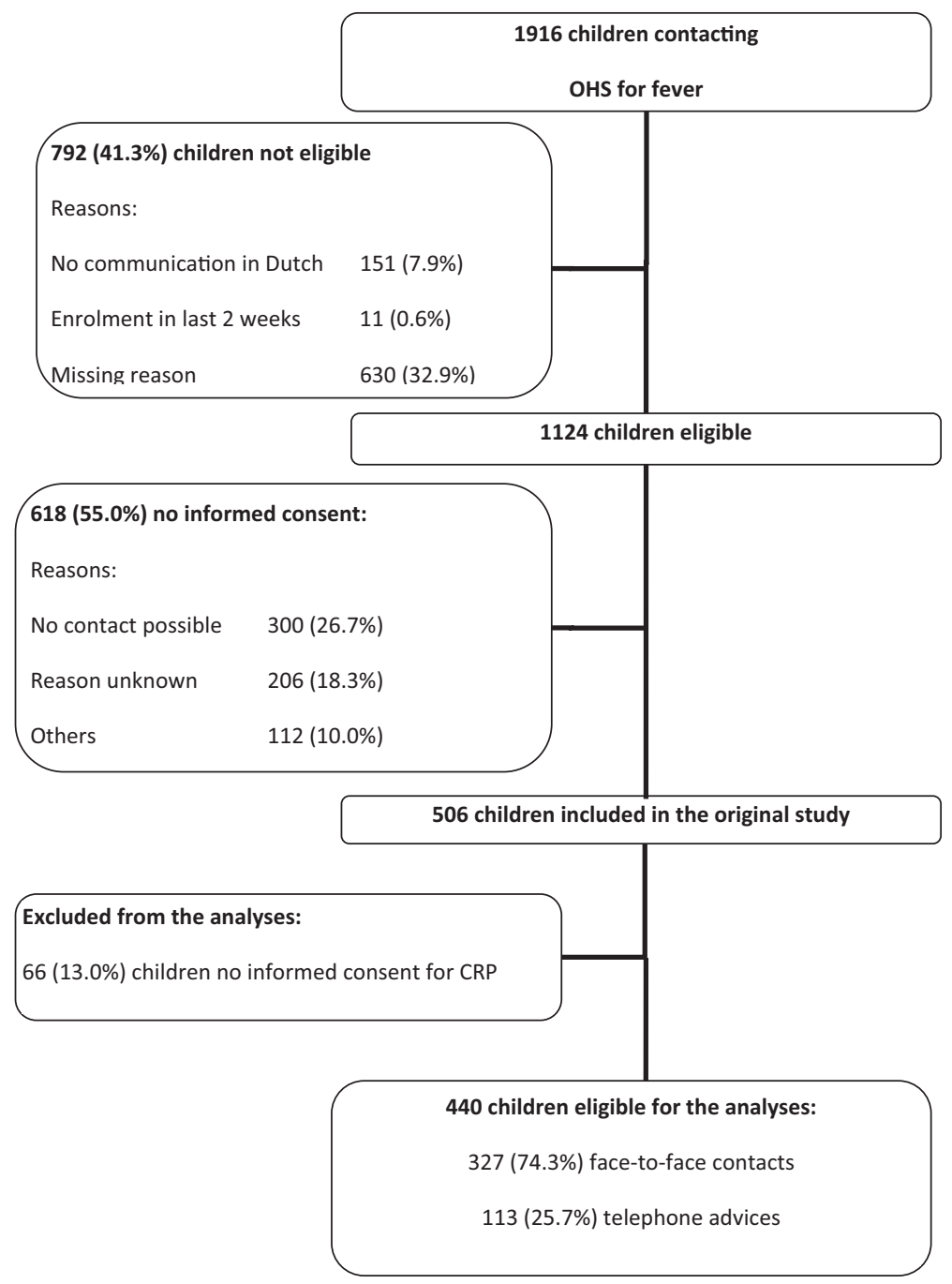

\section{Procedures}

In the Netherlands, patients should in principle first contact the General Practitioner Cooperative for out-of-hours primary care. When parents contacted the OHS (mostly by phone), the triage nurses performed their usual triage based on the practice guideline of the Dutch College of General Practitioners. ${ }^{12}$ According to this guideline, a child was invited for a face-to-face consultation if the child was $<3$ months old, was very ill, was rapidly deteriorating, drank less than half of their normal consumption, had a rash that occurred during fever, was crying inconsolably, had a change in skin color, had a change in breathing pattern, was moaning or had apnea, had relevant comorbidity, or had fever for $>3$ days or a fever that increased after a fever-free period. In addition, a child was seen when the parents showed agitation, aggression, or persistent anxiety. GPs were free to prescribe the treatment of their choice or to refer the child to a pediatric emergency department. GPs work according to the Dutch national guidelines, and adherence to these guidelines is quite high. The standard of the Dutch College of General Practitioners on children with fever advises referring a febrile child to secondary care if at least 1 alarming symptom is present. ${ }^{12}$ However, an earlier study showed that GPs overruled the guideline's advice (ie, decided not to refer the child) when a child had 1 or 2 alarming symptoms present. When $\geq 3$ alarming symptoms were present, nearly all children were referred. ${ }^{15}$

A child was triaged as "self-care advice" and received telephonic advice only if none of the above findings were present. ${ }^{14}$ 
A trained research nurse visited all children (regardless of their triage results) at home within 24 hours of inclusion (median, 14 hours; range, 5-21 hours). Using a structured questionnaire, the research nurses recorded demographic data, physician contacts, and prescribed antibiotics as reported by the parents. In addition, a standardized physical examination (including clinical features of alarming signs and rectal temperature) was performed. During this home visit, capillary blood was obtained to determine the CRP concentration. The records of the child's own GP were examined for diagnoses and referrals during the 7 days after inclusion. In the case of referral, CRP concentrations were extracted from pediatric records.

\section{Alarming Signs}

We defined 4 alarming signs. The choice of alarming signs was based on national and international guidelines and was defined before analyses. ${ }^{12,16}$ Alarming signs at physical examination were defined as present if at least 1 of the 4 criteria listed was found during physical examination: (1) "drowsiness" was defined as a poor or moderate alertness; (2) "abnormal circulation" was defined as a capillary refill $>2$ seconds, a poor or moderate peripheral circulation of the skin (skin color), or a tachycardia; (3) "shortness of breath" was defined as "chest indrawings," "nasal flaring," or an increased respiratory rate; and (4) "dehydration" was defined as a capillary refill $>2$ seconds or a sunken fontanel if the child was aged $\leq 1$ year $^{17}$ (see the Appendix).

\section{Antibiotic Prescription}

Antibiotic prescription was reported by the parents and defined as antibiotic prescription at presentation (either by the GP at the OHS or by a pediatrician after direct referral), antibiotic prescription before contact with the OHS, or antibiotic prescriptions after 1 week.

\section{Statistical Analyses}

Children with and without a CRP measurement were compared using a Student $t$ test or Pearson $\chi^{2}$ test, as appropriate.

We used 2 different cutoff values for CRP based on those recommended by Van den Bruel et $\mathrm{al}^{9}$ : $\leq 20 \mathrm{mg} / \mathrm{L}$ to exclude $\mathrm{SI}$ and $>80 \mathrm{mg} / \mathrm{L}$ to include SI. We constructed $2 \times 2$ contingency tables for the 2 cutoffs of CRP and SI to calculate sensitivity, specificity, positive likelihood ratio $(\mathrm{LR}+)$, and negative likelihood ratio (LR-). Probabilities with a $95 \%$ confidence interval (CI) were calculated using OpenEpi. ${ }^{18}$ Children with an SI at presentation were excluded from the analyses of SI during follow-up.

We tested whether alarming signs influenced the relation between CRP and SI. We assumed that antibiotics given before the measurement of CRP could influence the relationship between CRP and SI. We tested this assumption by evaluating the association between CRP and SI in children with antibiotic prescription before or at presentation and in children without antibiotic prescription before or at presentation.

We calculated a sample size given the following assumptions: a power of $80 \%$, an $\alpha$ of $0.05,11 \%$ SIs in the population, CRP $>20 \mathrm{mg} / \mathrm{L}$ in $50 \%$ of cases, CRP $>80 \mathrm{mg} / \mathrm{L}$ in $15 \%$ of cases, and an 2.5 odds ratio of having an SI in the case of an abnormal CRP. This resulted in sample sizes of 418 cases for a CRP cutoff of $20 \mathrm{mg} / \mathrm{L}$ and 481 cases for a CRP cutoff of $80 \mathrm{mg} / \mathrm{L}$.

\section{Missing Values}

Of the 440 children included in the analyses, no alarming signs were noted in 13 (3.0\%). In 12 of these 13 children, alarming signs were not noted because of admission to a hospital. Given the recommendation in the Dutch guideline to refer a child with alarming symptoms, ${ }^{12}$ we assumed that alarming signs were positive in those 12 admitted children.

\section{Results}

In the original study, 506 children were included. Of those, 66 children showed resistance to the finger prick, which for this analysis was defined as no informed consent from the child. Finally, a CRP concentration was available for 440 children. Table 1 presents comparisons between the children with and without a CRP measurement.

Of all 440 children, 34 (7.7\%) were referred and $16(3.6 \%)$ were diagnosed with a serious illness by the GP. Of the 50 children (11.4\%) at high risk for an SI, 30 children $(6.8 \% ; 18$ [4.1\%] referrals and 12 [2.7\%] with a diagnosis of a serious illness) were at high risk for an SI at presentation and 20 (4.5\%) developed an SI during follow-up (16 [3.6\%] referrals and $4[0.9 \%]$ with a diagnosis of a serious illness). The CRP concentrations ranged from $<7$ 
Table 1. Characteristics of Children with $(n=440)$ and without $(n=66)$ C-Reactive Protein Measurement (CRP)

\begin{tabular}{|c|c|c|}
\hline & $\begin{array}{l}\text { Children with CRP measured } \\
\qquad(\mathrm{n}=440)\end{array}$ & $\begin{array}{c}\text { Children without CRP measured } \\
\qquad(\mathrm{n}=66)\end{array}$ \\
\hline \multicolumn{3}{|l|}{ Age (months)* } \\
\hline Mean (SD) & $25(17)$ & $30(18)$ \\
\hline Median (range) & $20(3-70)$ & $28(3-66)$ \\
\hline Male sex & $254(57.7)$ & $35(53.0)$ \\
\hline Immigrant (yes) & $228(51.8)$ & $31(47.0)$ \\
\hline $\begin{array}{l}\text { Face-to-face contact with a general } \\
\text { practitioner at presentation }\end{array}$ & $327(74.3)$ & $44(66.7)$ \\
\hline Antibiotic prescriptions before presentation & $37(8.4)$ & $7(10.6)$ \\
\hline Referral at initial presentation $^{\dagger}$ & $18(4.1)$ & $8(12.1)$ \\
\hline Serious illness at presentation ${ }^{\ddagger}$ & $25(5.7)$ & $8(12.1)$ \\
\hline SI at presentation $\$$ & $30(6.8)$ & $10(15.2)$ \\
\hline Referral after 1 week & $34(7.7)$ & $8(12.1)$ \\
\hline Serious illness after 1 week & $39(8.9)$ & $8(12.1)$ \\
\hline SI after 1 week & $50(11.4)$ & $10(15.2)$ \\
\hline Antibiotic prescriptions after 1 week & $181 / 432(41.9)$ & $23 / 58(39.7)$ \\
\hline
\end{tabular}

Data are $\mathrm{n}(\%)$ unless otherwise indicated.

${ }^{*}$ Significant difference (mean difference, 5.7; 95\% confidence interval, 1.2-10.1).

${ }^{\dagger}$ Significant difference $\left(\chi^{2}=7.593 ; \mathrm{df}=1 ; P=0.006\right)$

${ }^{\ddagger}$ Significant difference $\left(\chi^{2}=3.904 ; \mathrm{df}=1 ; P=0.048\right)$

${ }^{\S}$ Significant difference $\left(\chi^{2}=5.474 ; \mathrm{df}=1 ; P=0.019\right)$.

$\mathrm{SD}$, standard deviation; SI, serious infection (defined as a serious illness or referral).

to $>251 \mathrm{mg} / \mathrm{L}$. Among all 440 children, CRP was $\leq 20 \mathrm{mg} / \mathrm{L}$ in $232(52.7 \%)$ and $>80 \mathrm{mg} / \mathrm{L}$ in 66 (15.0\%).

\section{CRP Concentrations to Rule in or Rule out SI}

For the characteristics of the CRP test for SI (either at presentation or during follow-up) or to predict an SI during follow-up, see Tables 2 and 3, respectively.

A CRP $>80 \mathrm{mg} / \mathrm{L}$ significantly increased the probability of an SI, from $11.4 \%$ (50 of 440 children; $95 \%$ CI, $8.7-14.7$ ) to $21.2 \%$ (14 of 66 children; $95 \% \mathrm{CI}, 13.1-32.5)$. A CRP $>80 \mathrm{mg} / \mathrm{L}$ at presentation increased the probability of an SI during follow-up from $4.9 \%$ (20 of 410 children; $95 \%$ CI, $3.2-7.4$ ) to $8.7 \%$ (5 of 57 children; $95 \%$ CI, 3.8-18.9; difference not significant). In both groups the LR+ was around $2.0(2.1$ [95\% CI, 1.3-3.5] and 1.9 [95\% CI, 0.8-4.2], respectively).

A CRP $\leq 20 \mathrm{mg} / \mathrm{L}$ did not change the probability of having no SI. In both groups the LR - was around 1.0.

\section{Alarming Signs}

Among all 440 children, the presence of alarming signs at physical examination was known in 439
(99.7\%). Of those 439 children, 205 (46.7\%) had at least 1 alarming sign during the physical examination; of those 205 children, 31 (15.1\%; 95\% CI, 10.9-20.7) had an SI. Of the 439 children, 234 (53.3\%) had no alarming signs at physical examination, and 18 of those $(7.7 \%$; $95 \%$ CI, $4.9-11.8)$ had an SI.

In children with alarming signs as well as in children without alarming signs there was no difference in the test characteristics of CRP $\leq 20$ and $>80 \mathrm{mg} / \mathrm{L}$. In children with or without alarming signs $(\mathrm{CRP} \leq 20 \mathrm{mg} / \mathrm{L}: \mathrm{LR}-=1.1$ [95\% CI, $0.8-$ 1.6] and 0.7 [95\% CI, 0.4-1.3], respectively; CRP $>80 \mathrm{mg} / \mathrm{L}: \mathrm{LR}+=1.7$ [95\% CI, 0.8-3.6] and 2.5 [95\% CI, 1.2-5.2], respectively).

\section{Antibiotic Prescriptions}

Of the 440 children, a record of whether antibiotics were prescribed was available for 415 children (94.3\%). Of those 415 children, 146 (33.2\%) received an antibiotic prescription (37 children before presentation, 109 children at presentation). Of the 146 children with an antibiotic prescription, 24 (16.4\%; 95\% CI, 11.3-23.3) had an SI (either at presentation or during follow-up). Among the 269 

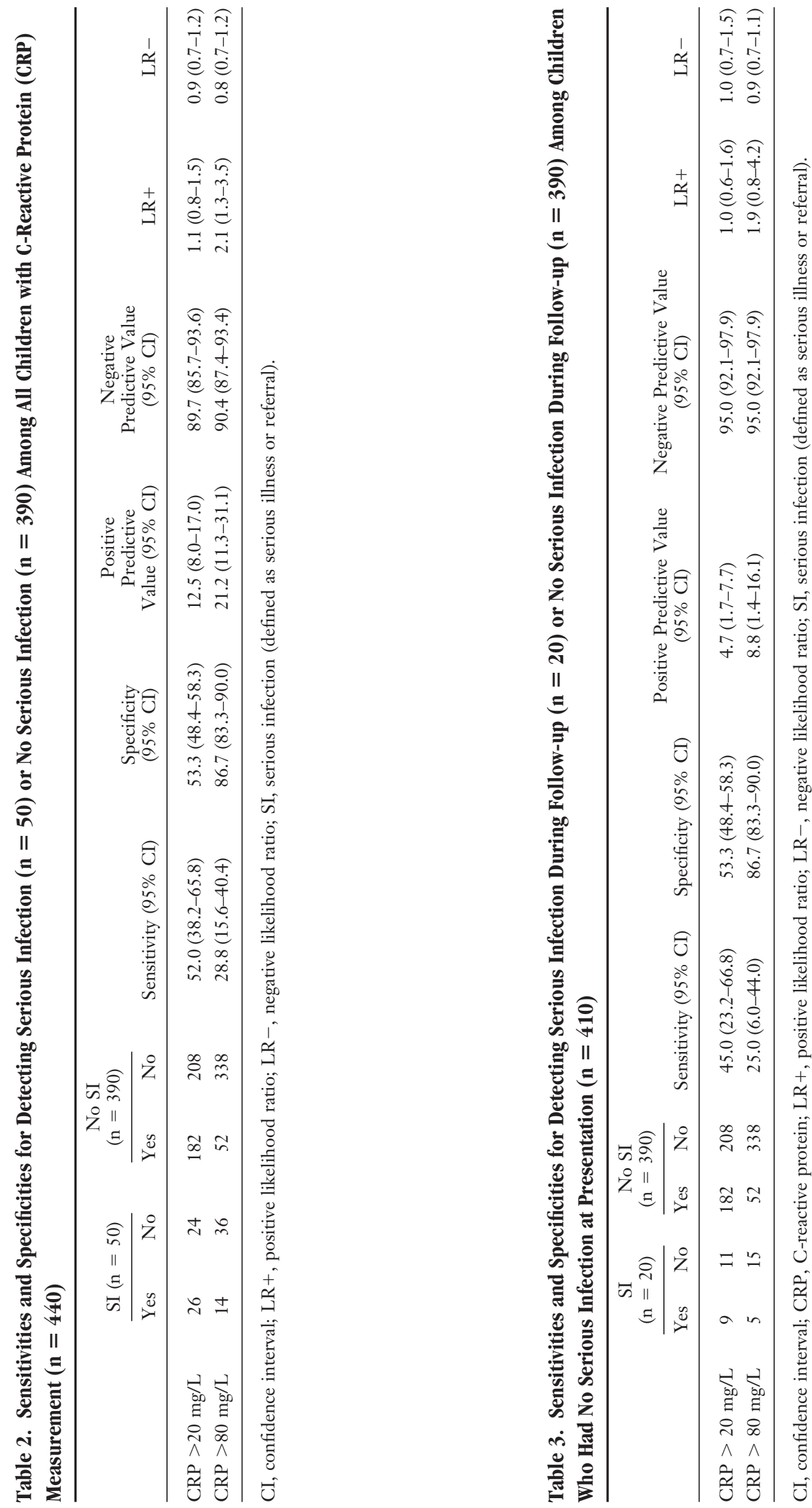
children without an antibiotic prescription, 25 had an SI (9.3\%; 95\% CI, 6.4-13.4).

There was no difference in the diagnostic value of CRP between children with or without antibiotics (CRP $>80 \mathrm{mg} / \mathrm{L}: \mathrm{LR}+=1.5$ [95\% CI, 0.73.2] and 2.2 [95\% CI, 1.0-4.7], respectively; CRP $<20 \mathrm{mg} / \mathrm{L}: \mathrm{LR}-=1.0$ [95\% CI, 0.6-1.6] and 1.0 [95\% CI, 0.7-1.4], respectively).

\section{Discussion}

\section{Summary of Main Findings}

CRP was measured in 440 children. A CRP $>80$ $\mathrm{mg} / \mathrm{L}$ increased the probability of an SI (either at presentation or during follow-up) from $11.4 \%$ (95\% CI, 8.7-14.7) to $21.2 \%$ (95\% CI, 13.1-32.5) (statistically significant), and of a SI during follow-up from 4.9\% (95\% CI, 3.2-7.4) to $8.7 \%$ (95\% CI, 3.8-18.9; difference not significant). In both these groups $\mathrm{LR}+$ was $\sim 2.0$. A CRP $\leq 20 \mathrm{mg} / \mathrm{L}$ was not able to preclude SI; in both groups the LR - was $\sim 1.0$.

Test characteristics of both CRP concentrations did not differ in children with and without alarming signs. Antibiotic use at the time of CRP measurement did not influence the association between CRP and the probability of an SI.

\section{Strengths and Limitations}

A strength of this study was that the outcome "at high risk of SI" was independent of the CRP concentration, since the for the GP and the research team were blinded to the CRP test results. Therefore the CRP results could not have influenced a diagnosis of an SI.

A total of 66 children from the original study gave no informed consent for the finger prick and were therefore excluded from this analysis. These children had a probability for SI that was similar to that of the included children. However, children without a CRP measurement were older (by 5.7 months overall) than the 440 included children. This difference might be because older children are more likely to offer resistance to a finger prick than younger children. In addition, we assumed that all referred children would have had an alarming symptom; if this was not the case, then this assumption would slightly overestimate the prevalence of SI among children with an alarming symptom. ${ }^{15} \mathrm{~A}$ similar effect in children without an alarming symptom will be negligible because of the small numbers involved.

The power calculation showed that for the CRP test cutoff values of 20 and $80 \mathrm{mg} / \mathrm{L}$, this study population was about large enough not to miss a serious (odds ratio $>2.5$ ) performance of the test if that exists.

Although the data date back to 2006, we assume that this has no effect on the diagnostic value of CRP to identify SI. Triage systems at Dutch OHSs are still basically the same. In addition, CRP is still not part of the standard care for febrile children in Dutch general practice.

\section{Comparisons with the Existing Literature}

A systematic review of the diagnostic value of laboratory tests to identify SI in febrile children examined 5 studies performed in an ambulatory setting (mainly referred children with intermediate to high prevalences of SI) and included children aged 1 month to 18 years. ${ }^{9}$ Their reported specificity for CRP $>80 \mathrm{mg} / \mathrm{L}$ was comparable to the specificity we found (86.7\% [95\% CI, 83.3-90.0] vs $>90 \%)$, ${ }^{9}$ but we found lower sensitivity (28.8\% [95\% CI, $15.6-40.4]$ vs $40 \%$ to $50 \%$ ). In our study the sensitivity and specificity for the cutoff CRP concentration of $20 \mathrm{mg} / \mathrm{L}$ (sensitivity: $52.0 \%$ [95\% CI, 38.2-65.8]; specificity: 53.3 [95\% CI, 48.4-58.3]) were much lower than those reported by Van den Bruel et $\mathrm{al}^{9}$ (sensitivity $>80 \%$, specificity $70 \%$ ). In our study the prevalence of SI of $11.4 \%$ is comparable to studies with an intermediate prevalence in the systematic review ("intermediate" was defined as $5 \%$ to $20 \%) .{ }^{9}$ Therefore, a difference in prevalence is not likely to explain the differences in the diagnostic values of CRP. Because we also included children who developed an SI during follow-up, the sensitivity in the cross-sectional studies included in the review might be overestimated.

We know that in children directly referred after face-to-face contact at the OHS $(\mathrm{n}=25)$, the observed risk of serious illness was $72 \%$, and $76 \%$ of the referred children were admitted to a hospital (data not shown). ${ }^{2}$ Therefore, misclassification of children without an SI as having an SI is estimated at around $30 \%$ to $25 \%$; this might have influenced our results. However, after restricting our definition of SI to "SI without referral," similar results were found (data not shown). 


\section{Relevance for Clinical Practice}

Using a value of CRP $\leq 20 \mathrm{mg} / \mathrm{L}$ to rule out SI, more than half of the children in need of medical care would have been missed (ie, 24 of 50 children, or $48.0 \%$ ), and CRP $>80 \mathrm{mg} / \mathrm{L}$ identified only 14 of the 50 children with an SI (28.0\%) (Table 2). In addition, in children without an SI at presentation, CRP had no clinically relevant predictive value for SI during follow-up (LR+, 1.9; 95\% CI, 0.8-4.2).

Together, these findings lead us to conclude that CRP has little clinically relevant value in the triage of febrile children, that is, in discriminating those in need of medical care from those who are not. However, the additional value of CRP in the management of febrile children needs further evaluation in randomized clinical trials.

The authors thank all the parents and children who participated in this study; the receptionists of the GP cooperative in Rotterdam-South; Berth J. Broekman (manager of the GP cooperative South); and Eef van Dijk, director of the central GP cooperatives Rijnmond.

\section{References}

1. Van den Bruel A, Bartholomeeusen S, Aertgeerts B, Truyers C, Buntinx F. Serious infections in children: an incidence study in family practice. BMC Fam Pract 2006;7:23.

2. Kool M. Serious infections and healthcare use in children with fever presenting at a general practice out-of-hours service. In: Febrile children at a general practice out-of-hours service [thesis]. Rotterdam: Erasmus University; 2015.

3. Cals JW, Butler CC, Hopstaken RM, Hood K, Dinant GJ. Effect of point of care testing for $\mathrm{C}$ reactive protein and training in communication skills on antibiotic use in lower respiratory tract infections: cluster randomized trial. BMC 2009;338:b1374.

4. Pepys MB, Hirschfield GM. C-reactive protein: a critical update. J Clin Invest 2003;111:1805-12.

5. Hopstaken RM, Muris JW, Knottnerus JA, et al. Contributions of symptoms, signs, erythrocyte sedimentation rate, and C-reactive protein to a diagnosis of pneumonia in acute lower respiratory tract infection. Br J Gen Pract 2003;53:358-64.

6. Melbye H, Straume B, Aasebo U, Brox J. The diagnosis of adult pneumonia in general practice. The diagnostic value of history, physical examination and some blood tests. Scand J Prim Health Care 1988;6: 111-7.

7. Melbye H, Straume B, Brox J. Laboratory tests for pneumonia in general practice: the diagnostic values depend on the duration of illness. Scand J Prim Health Care 1992;10:234-40.

8. Sanders S, Barnett A, Correa-Velez, Coulthard M, Doust J. Systematic review of the diagnostic accuracy of C-reactive protein to detect bacterial infection in nonhospitalized infants and children with fever. J Pediatr 2008;153:570-4.

9. Van den Bruel A, Thompson MJ, Hai-Hassan T, et al. Diagnostic value of laboratory tests in identifying serious infections in febrile children: systematic review. BMJ 2011;342:d3082.

10. Nabulsi M, Hani A, Karam M. Impact of C-reactive protein test results on evidence-based decision-making in cases of bacterial infection. BMC Pediatr 2012;12:140.

11. Monteny M, Ten Brinke MH, Van Brakel J, De Rijke YB, Berger MY. Point-of-care C-reactive protein testing in febrile children in general practice. Clin Chem Lab Med 2006;44:1428-32.

12. Berger MY, Boomsma LJ, Albeda FW, et al. The standard of the Dutch College of General Practitioners on children with fever. Huisarts Wet 2008;51: 287-96.

13. Dutch College of General Practitioners. [Dutch College of GPs' telephone guideline for triage and advice]. Utrecht: Nederlands Huisartsen Genootscha (NHG); 2002.

14. Kool M, Elshout G, Moll HA, Koes BW, van der Wouden JC, Berger MY. Duration of fever and course of symptoms in young febrile children presenting with uncomplicated illness. J Am Board Fam Med 2013;26:445-52.

15. Van Ierland Y, Elshout G, Moll HA, et al. Use of alarm features in referral of febrile children to the emergency department: an observational study. Br J Gen Pract 2014;64:e1-9.

16. National Collaborating Centre for Women's and Children's Health. Feverish illness in children; assessment and initial management in children younger than 5 years. 2nd ed. London: Royal College of Obstetricians and Gynaecologists Press; 2013.

17. Kool M. Reliability of parent-reported alarming symptoms in febrile children in general practice In: Febrile children at a general practice out-of-hours service [thesis]. Rotterdam: Erasmus University; 2015.

18. Dean AG, Sullivan KM, Soe MM. OpenEpi: Open Source Epidemiologic Statistics for Public Health, version 3.02a. Updated June 4, 2013. Available from: www.OpenEpi.com. Accessed November 1, 2014. 


\section{Appendix}

Tachycardia was defined as a pulse rate $\geq 160$ beats per minute (bpm) at age $\geq 1$ year, $\geq 150$ bpm at age 1 to 2 years, and $\geq 140 \mathrm{bpm}$ at age $\geq 2$ years. ${ }^{3}$ An increased respiratory rate was defined as $\geq 60$ breaths per minute at age 3 to 5 months, $\geq 50$ breaths per minute at age 6 to 12 months, and $\geq 40$ breaths per minute at age $\geq 12$ months.

These data are based on National Collaborating Center for Women's and Children's Health, "Feverish Illness in Children; Assessment and Initial Management in Children Younger Than 5 Years," 1st ed, London: Royal College of Obstetricians and Gynaecologists Press; 2007:1-16. 